Technische

Universität

Berlin

Qile Zhao, YinTong Wang, Shengfeng Gu, Fu Zheng, Chuang Shi, Maorong Ge, Harald Schuh

\title{
Refining ionospheric delay modeling for undifferenced and uncombined GNSS data processing
}

Document type

Journal article | Accepted version

(i. e. final author-created version that incorporates referee comments and is the version accepted for publication; also known as: Authors Accepted Manuscript (AAM), Final Draft, Postprint)

This version is available at

https://doi.org/10.14279/depositonce-11982

Citation details

Zhao, Q., Wang, Y., Gu, S., Zheng, F., Shi, C., Ge, M., \& Schuh, H. (2018). Refining ionospheric delay modeling for undifferenced and uncombined GNSS data processing. Journal of Geodesy, 93(4), 545-560. https://doi.org/10.1007/s00190-018-1180-9.

This is a post-peer-review, pre-copyedit version of an article published in Journal of Geodesy. The final authenticated version is available online at: https://doi.org/10.1007/s00190-018-1180-9.

Terms of use

This work is protected by copyright and/or related rights. You are free to use this work in any way that is permitted by the copyright and related rights legislation that applies to your use. For other uses you need to obtain permission from the rights-holder(s). 
Noname manuscript No.

(will be inserted by the editor)

Refining ionospheric delay modelling for undifferenced and uncombined GNSS data processing

Qile Zhao · YinTong Wang • Shengfeng Gu •

Fu Zheng · Chuang Shi · Maorong Ge •

Harald Schuh

Received: date / Revised version: date

Abstract To access the full capabilities of multi frequency signals from the modernized GPS, GLONASS and newly deployed BDS, Galileo, the undifferenced and uncombined observable model in which the individual signal of each frequency is treated as independent observable has drawn increasing interest in GNSS community. The ionosphere delay is the major issue in the undifferenced and uncombined observable model. Though several ionosphere delay parameterization approaches have been promoted, we argue that the functional model with only deterministic characteristic may not follow the irregular spatial and temporal variations. On the contrary, when the ionosphere delay is estimated as random walk or even white

Qile Zhao

GNSS Research Center, Wuhan University, 129 Luoyu Road, Wuhan 430079, China

YinTong Wang

GNSS Research Center, Wuhan University, 129 Luoyu Road, Wuhan 430079, China

Shengfeng $\mathrm{Gu}$

GNSS Research Center, Wuhan University, 129 Luoyu Road, Wuhan 430079, China

Shengfeng $\mathrm{Gu}$

Helmholtz Centre Potsdam, German Research Centre for Geosciences (GFZ), Telegrafenberg, 14473 Potsdam, Germany

Fu Zheng

GNSS Research Center, Wuhan University, 129 Luoyu Road, Wuhan 430079, China

Chuang Shi

GNSS Research Center, Wuhan University, 129 Luoyu Road, Wuhan 430079, China

Chuang Shi

School of Electronic and Information Engineering, Beihang University, 37 Xueyuan Road, Beijing 100083, China

Maorong Ge

Helmholtz Centre Potsdam, German Research Centre for Geosciences (GFZ), Telegrafenberg, 14473 Potsdam, Germany

Harald Schuh

Helmholtz Centre Potsdam, German Research Centre for Geosciences (GFZ), Telegrafenberg,

14473 Potsdam, Germany

*corresponding author: gsf@whu.edu.cn 
noise with only stochastic characteristic, the ionosphere terms turn out to be nonestimable or not sensitive to their absolute value. In the authors' previous study, we have developed the deterministic plus stochastic ionosphere model, denoted as DESIGN, in which the deterministic part expressed with second-order polynomial is estimated as piece-wise constant over 5 minutes and the stochastic part is estimated as random walk with constrains derived based on statistics of 4 weeks data in 2010. In this contribution, we further model the deterministic part with Fourier series and update the variogram of the stochastic part accordingly based on two year data collected by about 150 stations. From the statistic studies, it is concluded that the main frequency components are identical for different coefficients, different stations, as well as different ionosphere activity status, but with varying amplitude. Thus, in the Fourier series expression of the deterministic part, we fix the frequency and estimate the amplitude as daily constant unknowns. Concerning the stochastic component, the variation of variogram is both, geomagnetic latitude and ionosphere activity status dependent. Thus, we use the Gaussian function and Epstein function to model the variation of geomagnetic latitude and ionosphere activity status, respectively. Based on the undifferenced and uncombined observable model with ionosphere constrained with DESIGN, both dual frequency and single frequency PPP are carried out to demonstrate its efficiency with three months? data collected in 2010, 2014, and 2017 with different ionosphere activity status. The experimental results suggest, that compared with Ionosphere-Free model and our previous method, the averaged 3D improvement of our new method is $17.8 \%$ and $7.6 \%$ for dual frequency PPP, respectively. While for single frequency PPP, the averaged $3 \mathrm{D}$ improvement is $37.0 \%$ and $14 \%$, respectively.

Keywords Undifferenced and uncombined observable, Ionosphere delay, DESIGN, Precise Point Positioning

\section{Introduction}

The ionosphere is a layer of plasma that surrounds the earth in the altitude range from about $50 \mathrm{~km}$ to $1500 \mathrm{~km}$. It exerts a refraction effect on electromagnetic signals, e.g., GNSS when traveling through. This effect will cause range errors that may reach up to about 100 meters for low elevation angles [Schaer (1999)]. For high precision GNSS navigation and positioning applications, the ionosphere effect needs to be carefully eliminated in the data processing [YiChung (1997), Shi et al. (2012)].

Typically, by analyzing the dispersive characteristic of the ionosphere effects, the so called Ionosphere Free (IF) combination forms the basic observable under the dual-frequency environment [Zumberge et al. (1997), Kouba and Héroux (2001), Shi et al. (2012B)]. However, with signals transmitted on more than two frequencies from the modernized GPS and GLONASS, as well as the newly deployed satellite systems, i.e., BDS and Galileo, the undifferenced and uncombined data processing strategy to avoid any combination has begun to receive increasing interests within the GNSS community [Schönemann et al. (2011), Gu et al. (2013)]. The fundamentally new aspect of such a processing strategy is that all undifferenced and uncombined available signals from variety of frequencies of multi-GNSS are incorporated in a single parameter estimation system directly, regardless the 
type of the receiver [Gu et al. (2013)]. Among other difficulties, the ionosphere delay should be estimated as unknown simultaneously with the other parameters in this model. Obviously, the performance of the novel algorithm mainly depends on the capability of the particular ionospheric parameterization method involved.

The first systematic study of GNSS ionosphere parameterization can be found in the International GNSS Service (IGS) context. Since 1998, IGS has established the Ionosphere Working Group (Iono-WG) with the intention to continually monitor the ionosphere [Schaer (1999),Hernández-Pajares et al. (2009)]. Since then, individual Global Ionosphere Maps (GIM) in IONEX format were independently generated on a regular basis by different Ionospheric Associate Analysis Centers (IAACs), including Center for Orbit Determination in Europe (CODE; University of Berne, Switzerland), Jet Propulsion Laboratory (JPL; Pasadena, CA, USA), European Space Operations Center of European Space Agency (ESOC; Darmstadt, Germany), and Technical University of Catalonia/gAGE (UPC; Barcelona, Spain) [Li et al. (2012)]. More recently, three institutes, i.e., Natural Resources of Canada (NRCan; Canada), Institute of Geodesy and Geophysics (IGG; Chinese Academy of Sciences) and Wuhan University (WHU; China) are accepted as new IAACs according to IGS 2016 Workshop. Under the umbrella of the IGS Iono-WG, the final IGS ionosphere product is derived by combining Total Electron Content (TEC) maps of different AC by using weights computed by the Validation Center UWM (University of Warmia and Mazury; Poland), in order to get a more accurate product.

Attributed to the efforts of IGS Iono-WG, various models are available nowadays to approximate the temporal-spatial behavior of ionosphere delay over the coverage area. Specifically, the sphere harmonic function (SHF) is employed by CODE by noticing the deterministic property of ionosphere delay across spatial domain [Schaer (1999)]. While, taking its stochastic property into consideration, [Blanch J (2003)] developed an ionospheric estimation algorithm based on Kriging. However, since these methods are originally formulated for wide area ionosphere modeling only, it can hardly adapt itself to the undifferenced and uncombined observable processing model.

Alternatively, a simple and straight-forward way to model ionosphere delay in GNSS data processing is to estimate one parameter for each station-satellite pair. In the case of sing-frequency observable this approach is exactly equivalent to the IF combination solution, providing that the ionosphere are estimated as white noise epoch wise. Note that the conclusion is not hold for dual-frequency since the ionospheric delays on the code and carrier-phase observable are individually eliminated during the code and carrier-phase IF combination. [Bock et al. (2009)] has tried to use random walk for representation of each ionospheric delay parameter in the time domain. Since no spatial constrains are imposed across different LOS (Line of Sight) ionosphere delays, the ionosphere parameter in the study of [Bock et al. (2009)] is actually inseparable from the satellite Differential Code Bias (DCB). To overcome this problem, polynomial and trigonometric series are commonly accepted as spatial functions for local ionosphere [Chen and Gao (2005)]. Unfortunately, these models do not capture small scale and high frequency ionospheric disturbances well. Consequently, [Yuan and Ou (2004)] proposed the Generalized Trigonometric Serial Functions (GTSF) by adaptive parameter set with some preliminary results. In recent years, [Li et al. (2012)] and [Shi et al. (2016)] adapted this GTSF method to model the local ionospheric vertical TEC of each 
individual station in the BDS DCB estimation. It should be noted, that the GTSF model is actually only applied in the second step for the separation of ionosphere delay and satellite DCB, but not satisfy the uncombined PPP data processing with an accuracy of center-meter level. [Shi et al. (2012)] argued, that one of the crucial factors in high precision ionosphere parameterization is that both deterministic and random properties of the ionosphere delay should be taken into consideration, and proposed an elaborated ionospheric parameterization methods for undifferenced and uncombined observable data processing. Though the proposed formula of [Shi et al. (2012)] has been demonstrated as an efficient way to describe ionospheric variations for PPP with both single- and dual-frequency receivers [Lou et al. (2015)], its functional part expressed by a second-order polynomial is casually treated as piece-wise constant over 5 minute.

From the above discussion, it is concluded that there are only few studies that investigate the high precision ionosphere delay parameterization method to improve the performance of solutions with undifferenced and uncombined GNSS data. Actually, this contribution is a subsequent research of the authors' previous studies, thus, the readers are highly recommended to have a review of [Shi et al. (2012)], [Gu et al. (2013)] and [Lou et al. (2015)]. In this contribution, based on the undifferenced and uncombined observable model, the shortage of traditional functional or stochastic only ionosphere parameterization approaches are discussed. In addition, our previous studies are also analyzed. Then the ionosphere model of [Shi et al. (2012)] are refined based on Fourier and stochastic analysis. To verify the efficiency of the new ionosphere formula, we adopt it in the undifferenced and uncombined data processing model and finally carried out the numerical demonstration with over three months observable under different ionosphere activity status in terms of dual frequency and single frequency multi-GNSS PPP.

\section{Notation}

In this paper, we adopt the following conventions: the satellite system identifiers $(G R C E)$ as suggested in RINEX 3.02 format are used to denote GPS, GLONASS, BDS and Galileo, respectively. Matrices and vectors are denoted in bold form, while scalars are denoted in regular form. And the term in its bold form stands for the corresponding vector, e.g., $\boldsymbol{P}_{\boldsymbol{r}}=\left(\begin{array}{llll}\widetilde{P}_{r}^{1} & \widetilde{P}_{r}^{2} \ldots \widetilde{P}_{r}^{j}\end{array}\right)^{T}$ and $\boldsymbol{t}=$ $\left(t^{1} t^{2} \cdots t^{j}\right)^{T}$ are the pseudo-range observation minus calculated (OMC) vector for receiver $r$ and satellite clock vector for all the $j$ satellites. For more details concerning these vectors, we refer to subsection 3.1. In addition, a few notations are defined for future reference:

$$
\begin{aligned}
\boldsymbol{z}_{\boldsymbol{s}} & =\left(\begin{array}{llll}
0 & 0 & \cdots & 0
\end{array}\right)^{T} \\
\boldsymbol{u}_{\boldsymbol{s}} & =\left(\begin{array}{llll}
1 & 1 & \cdots & 1
\end{array}\right)^{T} \\
\boldsymbol{Z}_{\boldsymbol{s}} & =\left(\begin{array}{lll}
0 & \cdots & 0 \\
0 & \ddots & 0 \\
0 & \cdots & 0
\end{array}\right)
\end{aligned}
$$




$$
\boldsymbol{U}_{\boldsymbol{s}}=\left(\begin{array}{ccc}
1 & \cdots & 0 \\
0 & \ddots & 0 \\
0 & \cdots & 1
\end{array}\right)
$$

as defined, $\boldsymbol{z}_{\boldsymbol{s}}$ is a $s \times 1$ vector with zero entries and $\boldsymbol{u}_{\boldsymbol{s}}$ is a $s \times 1$ vector with one entries, while $\boldsymbol{Z}_{\boldsymbol{s}}$ is a $s \times s$ matrix with zero entries and $\boldsymbol{U}_{\boldsymbol{s}}$ is a $s \times s$ identity matrix. And the dimensions and lengths of such vectors will generally be obvious from context. $E(\cdot)$ denotes the expectation operator, while $D(\cdot)$ denotes the covariance function.

\section{Methods}

3.1 Basic observable

The undifferenced and uncombined observable of the GNSS pseudo-range and carrier-phase are generally expressed as

$$
\left.\begin{array}{rl}
P_{r, f}^{s} & =\rho_{r}^{s}+t_{r}-t^{s}+\alpha_{r}^{s} \cdot T_{z}+b_{r, f}^{s} \\
& +\beta_{f} \cdot I_{r}^{s}+\varepsilon_{P} \\
\Phi_{r, f}^{s} & =\rho_{r}^{s}+t_{r}-t^{s}+\alpha_{r}^{s} \cdot T_{z}+b_{r, f}^{s}-N_{r, f}^{s} \\
& -\beta_{f} \cdot I_{r}^{s}+\varepsilon_{\Phi}
\end{array}\right\}
$$

in which, $P_{r, f}^{s}, \Phi_{r, f}^{s}$ are pseudo-range and carrier-phase from receiver $r$ to satellite $s(s=1, \cdots, j)$ on frequency $f(f=1, \cdots, k)$ in length units, respectively; $\rho$ is the geometric distance, while antenna phase center corrections as well as the phase windup should be applied to $P, \Phi$ before $\rho$ becomes unassociated with the frequency; $t\left(\begin{array}{c}s \\ r\end{array}\right)$ is the receiver and satellite clock error in length units respectively; $T_{z}$ is the zenith tropospheric delay that can be converted to slant with the mapping function $\alpha$; $I$ denotes the LOS TEC with the frequency dependent factor $\beta_{f}=40.3 / f^{2} ; b_{r}^{s}=b_{r}-b^{s}$ is the frequency dependent signal delay; $N$ is the float ambiguity in length units.

In addition, with the intention of discussion on ionosphere and satellite DCB parameters, it is assumed that the terms $\rho, t^{s}$ and $T_{z}$ are exactly known and have been applied to $\widetilde{P}$, $\widetilde{\Phi}$, and only dual-frequency, i.e., $k=2$ is involved. The assumption is promoted for the following discussion only since $t^{s}$ and $T_{z}$ are frequency independent, thus can be separated from ionosphere and satellite DCB parameters. Under the assumption, the observation equations based on Eq. (5) reads

$$
\left.\begin{array}{lr}
\widetilde{P}_{1}^{s}=t+b_{1}^{s} & +\beta_{1} \cdot I^{s}+\varepsilon_{P} \\
\widetilde{P}_{2}^{s}=t+b_{2}^{s} & +\beta_{2} \cdot I^{s}+\varepsilon_{P} \\
\widetilde{\Phi}_{1}^{s}=t+b_{1}^{s}-N_{1}^{s} & -\beta_{1} \cdot I^{s}+\varepsilon_{\Phi} \\
\widetilde{\Phi}_{2}^{s}=t+b_{2}^{s}-N_{2}^{s} & -\beta_{2} \cdot I^{s}+\varepsilon_{\Phi}
\end{array}\right\}
$$

in which the receiver subscript $r$ is omitted for simplification. And $\widetilde{P}_{r}^{s}$ and $\widetilde{\Phi}_{r}^{s}$ are the OMC for pseudo-range and carrier phase, respectively, i.e., $\widetilde{P}_{r}^{s}=P_{r}^{s}-\rho_{r}^{s}$ and $\widetilde{\Phi}_{r}^{s}=\Phi_{r}^{s}-\rho_{r}^{s}$.

In the case that the receiver $r$ tracked $j$ satellites simultaneously, we have undifferenced and uncombined observable model in the matrix-vector form based on Eq. (6) for a single epoch as follow:

$$
\left(\begin{array}{llll}
\boldsymbol{P}_{\mathbf{1}} & \boldsymbol{P}_{\mathbf{2}} & \boldsymbol{\Phi}_{\mathbf{1}} & \boldsymbol{\Phi}_{\mathbf{2}}
\end{array}\right)^{T}=\boldsymbol{A}_{\boldsymbol{e}} \cdot\left(\begin{array}{llllll}
t & \boldsymbol{b}_{\mathbf{1}} & \boldsymbol{b}_{\mathbf{2}} & \boldsymbol{N}_{\mathbf{1}} & \boldsymbol{N}_{\mathbf{2}} \boldsymbol{I}
\end{array}\right)^{T}
$$


in which

$$
\boldsymbol{A}_{\boldsymbol{e}}=\left(\boldsymbol{u}_{\boldsymbol{4} j}\left(\begin{array}{l}
\boldsymbol{U}_{\mathbf{2 j}} \\
\boldsymbol{U}_{\mathbf{2} j}
\end{array}\right)\left(\begin{array}{c}
\boldsymbol{Z}_{\mathbf{2}} \\
\boldsymbol{U}_{\mathbf{2} j}
\end{array}\right)\left(\begin{array}{c}
\beta_{1} \cdot \boldsymbol{U}_{j} \\
\beta_{2} \cdot \boldsymbol{U}_{j} \\
-\beta_{1} \cdot \boldsymbol{U}_{j} \\
-\beta_{2} \cdot \boldsymbol{U}_{j}
\end{array}\right)\right)
$$

Since $t_{r, f}^{s}$ and $b_{r, f}^{s}$ are linear dependent, it is assumed that

$$
\left.\begin{array}{l}
0=b_{r, 1} \\
0=b_{1}^{s}
\end{array}\right\}
$$

to make it uniquely solvable. The $r+s$ constrains are introduced to separate clocks from code biases for receiver and satellite, respectively. And it was proven that, in this case, $b_{r, 2}^{s}$ and $b_{r, 3}^{s}$ are actually known as DCB in IGS community [Gu et al. (2013),?]. Furthermore, to separate the satellite DCB from the receiver $\mathrm{DCB}$ in $b_{r}^{s}$, the zero-mean condition for all satellites is commonly adopted as suggested by [Feltens and Schaer(1998)]. In this case, the parameter vector $\boldsymbol{b}_{\mathbf{1}}$ is eliminated from Eq. (7) and Eq. (8) is expressed as

$$
\boldsymbol{A}_{\boldsymbol{e}}=(\underbrace{\boldsymbol{u}_{4 j}}_{\boldsymbol{A}_{1, e}} \underbrace{\left(\begin{array}{c}
Z_{j} \\
\boldsymbol{U}_{j} \\
\boldsymbol{Z}_{j} \\
\boldsymbol{U}_{j}
\end{array}\right)\left(\begin{array}{c}
Z_{2 j} \\
\boldsymbol{U}_{2 j}
\end{array}\right)}_{\boldsymbol{A}_{2, e}} \underbrace{\left(\begin{array}{c}
\beta_{1} \cdot \boldsymbol{U}_{j} \\
\beta_{2} \cdot \boldsymbol{U}_{j} \\
-\beta_{1} \cdot \boldsymbol{U}_{j} \\
-\beta_{2} \cdot U_{j}
\end{array}\right)}_{\boldsymbol{A}_{3, e}})
$$

Regarding the solution of Eq. (10), the necessary and sufficient condition for a system to be invertible lies in the fact that $\boldsymbol{A}_{\boldsymbol{e}}$ has the full column rank [Welsch (1979)]. However, a detailed analysis reveals the linear dependence between the columns as evidenced by

$$
\begin{aligned}
& 0=\left(\begin{array}{lll}
A_{1, e} & A_{2, e} & A_{3, e}
\end{array}\right) \cdot \omega^{T} \\
& \boldsymbol{\omega}=(\underbrace{-\beta_{1}}_{\boldsymbol{\omega}_{1}} \underbrace{\left(\beta_{1}-\beta_{2}\right) \cdot \boldsymbol{u}_{\boldsymbol{j}}^{T} 2 \beta_{1} \cdot \boldsymbol{u}_{\boldsymbol{j}}^{T} 2 \beta_{2} \cdot \boldsymbol{u}_{\boldsymbol{j}}^{T}}_{\boldsymbol{\omega}_{\mathbf{2}}} \underbrace{\boldsymbol{u}_{j}^{T}}_{\boldsymbol{\omega}_{\mathbf{3}}})
\end{aligned}
$$

the notation $\underbrace{\sim}$ in Eq. (10) and Eq. (11) refers to the alias of the corresponding sub-matrix.

Suppose that there are totally $k$ epochs data collected, then the accumulated design matrix $\boldsymbol{A}$ based on $\boldsymbol{A}_{\boldsymbol{e}}$ is written as

$$
\begin{aligned}
& \boldsymbol{A}=\left(\begin{array}{lll}
A_{1} & A_{2} & A_{3}
\end{array}\right)
\end{aligned}
$$

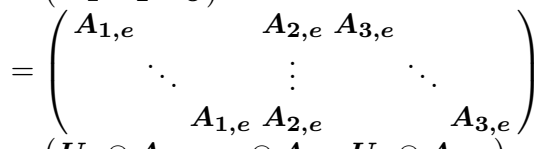

$$
\begin{aligned}
& =\left(\boldsymbol{U}_{\boldsymbol{k}} \otimes \boldsymbol{A}_{\mathbf{1}, \boldsymbol{e}} \boldsymbol{u}_{\boldsymbol{k}} \otimes \boldsymbol{A}_{\mathbf{2}, \boldsymbol{e}} \boldsymbol{U}_{\boldsymbol{k}} \otimes \boldsymbol{A}_{\mathbf{3}, \boldsymbol{e}}\right)
\end{aligned}
$$

in which, the ionosphere delays are treated as white noise, and the DCB and ambiguity terms are estimated as constant. Obviously, since the coefficients keep 
unchanged over the experiment period, the relation still holds true by extending Eq. (11)

$$
0=\left(\boldsymbol{U}_{\boldsymbol{k}} \otimes \boldsymbol{A}_{1, e} \boldsymbol{u}_{\boldsymbol{k}} \otimes \boldsymbol{A}_{2, e} \boldsymbol{U}_{\boldsymbol{k}} \otimes \boldsymbol{A}_{3, e}\right) \cdot\left(\begin{array}{c}
\boldsymbol{\omega}_{1}^{T} \\
\ddots \\
\boldsymbol{\omega}_{1}^{T} \\
\boldsymbol{\omega}_{2}^{T} \\
\omega_{3}^{T} \\
\ddots \\
\omega_{3}^{T}
\end{array}\right)
$$

or

$$
0=\left(\begin{array}{lll}
A_{1} & A_{2} & A_{3}
\end{array}\right) \cdot\left(\boldsymbol{u}_{\boldsymbol{k}}^{T} \otimes \boldsymbol{\omega}_{\mathbf{1}} \boldsymbol{\omega}_{\mathbf{2}} \boldsymbol{u}_{\boldsymbol{k}}^{T} \otimes \boldsymbol{\omega}_{\mathbf{3}}\right)^{T}
$$

which indicates that without external constrains, the ionospheric term $\boldsymbol{I}$ is a nonestimable parameter even with accumulated measurements. Numerically speaking, a singular problem can be solved by either generalized inverse [Rao and Mitra (1971)] or regularization of the system with a set of constrains [Blaha (1982)]. These two techniques are equivalent since each choice of generalized inverse corresponds to a choice of regularization constrains, and both lead to identical result. In fact, such an algorithm leads to a solution class, among which, one solution can be converted to another theoretically through S-transformation [Baarda (1981)]. However, as far as ionospheric parameters estimation are concerned, this problem is more complicated since only when the constrains attached ideally describe the ionosphere spatial and temporal variations, we can regard these solutions as unbiased estimations of the "absolute" ionospheric delays. Otherwise, the estimations are biased due to mismodeling or only "relative" values are obtained due to the datum deficiency [Gu et al. (2013)].

\subsection{Existing Ionospheric Parameterization Approaches}

\subsubsection{GTSF}

It is well known that the temporal-spatial behavior of ionosphere delay can be approximated by a set of functions, i.e., polynomial functions (POLY) or trigonometric series functions (TSF). Furthermore, these functions have been generalized by [Yuan and $\mathrm{Ou}(2004)$ ] with an adaptive parameter set over a single-day period, denoted as GTSF

$$
\left.\begin{array}{rl}
I^{s} & =M \cdot I_{z}^{s}(\varphi, \lambda) \\
I_{z}^{S}(\varphi, \lambda) & =\sum_{n=0}^{n_{\max }} \sum_{m=0}^{m_{\max }}\left\{E_{n \cdot m} \cdot\left(\varphi-\varphi_{0}\right)^{n} \cdot \lambda^{m}\right\} \\
& +\sum_{k=0}^{k_{\max }}\left\{C_{k} \cdot \cos (k \cdot \lambda)+S_{k} \cdot \sin (k \cdot \lambda)\right\} \\
\lambda & =\frac{2 \pi \cdot(t-14)}{24}
\end{array}\right\}
$$

where $I_{z}^{s}$ is the zenith ionosphere delay that can be convented to LOS with the mapping function $M ; \varphi$ and $\varphi_{0}$ are the geomagnetic latitude of ionospheric pierce point (IPP) and the receiver, respectively; $\lambda$ is the solar longitude of IPP, that 
refers to the local mean maximum of ionization of about 14:00; $t$ is the local time at IPP; $n_{\max }, m_{\max }$ are the maximum degree of the polynomial coefficient; $k_{\max }$ is the maximum degree of the Fourier series; $E_{n m}, C_{k}$ and $S_{k}$ are the GTSF coefficients.

Denoting

$$
\begin{aligned}
& A_{G T S F, e}= \\
& \left(M^{1} \ldots M^{j}\right) \cdot\left(\begin{array}{llllll}
\boldsymbol{A}_{\boldsymbol{E}}^{1} & \boldsymbol{A}_{\boldsymbol{C}}^{1} & \boldsymbol{A}_{\boldsymbol{S}}^{1} & & & \\
& & \ddots & & \\
& & & & \\
& & & \boldsymbol{A}_{\boldsymbol{E}}^{j} & \boldsymbol{A}_{\boldsymbol{C}}^{j} & \boldsymbol{A}_{\boldsymbol{S}}^{j}
\end{array}\right)
\end{aligned}
$$

with $\boldsymbol{A}_{\boldsymbol{E}}^{s}, \boldsymbol{A}_{\boldsymbol{C}}^{s}$ and $\boldsymbol{A}_{\boldsymbol{C}}^{s}$ the coefficient vectors for $E_{n \cdot m}, C_{k}$ and $S_{k}$, respectively, i.e.,

$$
\begin{aligned}
& \left(\begin{array}{c}
\boldsymbol{A}_{\boldsymbol{E}}^{s} \\
\boldsymbol{A}_{\boldsymbol{C}}^{s} \\
\boldsymbol{A}_{\boldsymbol{C}}^{s}
\end{array}\right)= \\
& \left(\begin{array}{ccccc}
1 & \left(\varphi-\varphi_{0}\right) & \lambda & \cdots & \left(\varphi-\varphi_{0}\right)^{n_{\max }} \cdot \lambda^{m_{\max }} \\
1 & \cos (\lambda) & \cos (2 \cdot \lambda) & \cdots & \cos \left(k_{\max } \cdot \lambda\right) \\
0 & \sin (\lambda) & \sin (2 \cdot \lambda) & \cdots & \sin \left(k_{\max } \cdot \lambda\right)
\end{array}\right)
\end{aligned}
$$

where the subscript ${ }^{s}$ for different satellite accompany with $\varphi$ and $\lambda$ is omitted for simplification. Then we have the constrains

$$
0=A_{c s t} \cdot\left(I E_{n \cdot m} C_{k} S_{k}\right)
$$

with

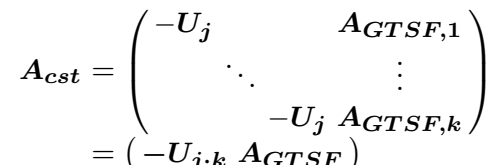

in which it is noted that, the matrix $\boldsymbol{A}_{\boldsymbol{G T S F}, \boldsymbol{e}}$ is varied with the geometry of differ-

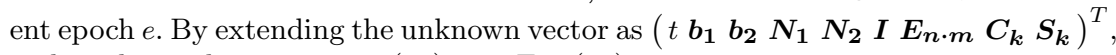
and applying the constrains (18) into Eq. (12), it comes

$$
A=\left(\begin{array}{rrrr}
A_{1} & A_{2} & A_{3} & 0 \\
& A_{\text {cst }}
\end{array}\right)
$$

By comparing Eq. (13) and Eq. (20), it is stated that to have the columns of $\boldsymbol{A}$ in Eq. (20) linear dependent, there should exist a vector $\boldsymbol{b}$ satisfying

$$
0=\left(\begin{array}{ll}
-\boldsymbol{U}_{j \cdot k} & A_{G T S F}
\end{array}\right) \cdot\left(\begin{array}{c}
\boldsymbol{u}_{\boldsymbol{k}} \otimes \boldsymbol{\omega}_{3}^{T} \\
\boldsymbol{b}
\end{array}\right)
$$

i.e.,

$$
u_{k} \otimes \omega_{3}^{T}=A_{G T S F} \cdot b
$$

Suppose that there are more rows than columns for $\boldsymbol{A}_{\boldsymbol{G T S F}}$, i.e., $(j \cdot k)>\left(n_{\max }\right.$. $\left.m_{\max }+2 \cdot k_{\max }\right)$, and attributed to the different geographical coordinates associated with the entries in $\boldsymbol{A}_{\boldsymbol{G T S F} \boldsymbol{T}, \boldsymbol{e}}$, which implies that the rows are independent, then Eq. (21) is overdetermined. Thus, the vector $\boldsymbol{b}$ does not exist, or in another word, 
the datum deficiency of Eq. (20) has been regularized by applying the constrains (12) with enough measurements.

By adjusting and selecting different types of model parameters, [Yuan and Ou (2004)] argued, that the different local ionospheric properties can be comparatively well described based on Eq. (12). However, the algorithm of parameter selection is still left unsolved so far. Moreover, though GTSF has been demonstrated as an efficient way in satellite DCB estimation [Li et al. (2012), Shi et al. (2016)], it failed to describe the ionospheric delay behavior with an accuracy of centimeter level.

3.2.2 $S I P$

Another popular ionosphere parameterization method is the so called Stochastic Ionosphere Parameter (SIP) [Schaer (1999), Bock et al. (2009)], in which the slant ionosphere delay $\boldsymbol{I}$ is treated as random walk, i.e., $\boldsymbol{I}_{\boldsymbol{i}+\mathbf{1}}=\boldsymbol{I}_{\boldsymbol{i}}+\boldsymbol{\omega}$ with $\omega \sim N\left(0 \boldsymbol{D}_{\boldsymbol{\omega}}\right)$, thus we have

$$
0=\boldsymbol{R}_{\boldsymbol{\omega}} \cdot \boldsymbol{I}_{\boldsymbol{i}+\mathbf{1}}-\boldsymbol{R}_{\boldsymbol{\omega}} \cdot \boldsymbol{I}_{\boldsymbol{i}}
$$

by writing the observation error $\boldsymbol{D}_{\boldsymbol{\omega}}^{-1}=\boldsymbol{P}_{\boldsymbol{\omega}}=\boldsymbol{R}_{\boldsymbol{\omega}}^{T} \cdot \boldsymbol{R}_{\boldsymbol{\omega}}$. Applying the constrains (23) into Eq. (12)

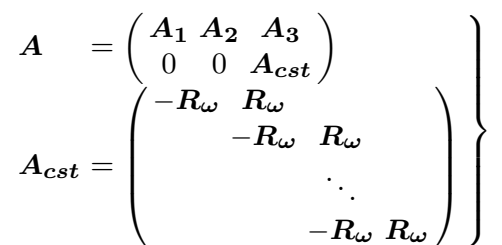

By substituting Eq. (24) into Eq. (14), it can be found that the ionosphere delay is still a non-estimable parameter as

$$
0=\left(\begin{array}{ccc}
\boldsymbol{A}_{1} & \boldsymbol{A}_{\mathbf{2}} & \boldsymbol{A}_{\mathbf{3}} \\
0 & 0 & \boldsymbol{A}_{\boldsymbol{c s t}}
\end{array}\right) \cdot\left(\begin{array}{llll}
\boldsymbol{u}_{\boldsymbol{k}}^{T} \otimes \boldsymbol{\omega}_{\mathbf{1}} & \boldsymbol{\omega}_{\mathbf{2}} & \boldsymbol{u}_{\boldsymbol{k}}^{T} \otimes \boldsymbol{\omega}_{\mathbf{3}}
\end{array}\right)^{T}
$$

Similarly, we can treat the zenith ionosphere delay $\boldsymbol{I}_{\boldsymbol{z}}$ as random walk. Then, for each epoch $e$, denoting $\frac{1}{\boldsymbol{M}^{i}}=\left(\frac{1}{M^{1}} \cdots \frac{1}{M^{j}}\right)^{T}$ the mapping function to convert $\boldsymbol{I}$ to $\boldsymbol{I}_{\boldsymbol{z}}$, Eq. (23) can be rewritten as

$$
0=\boldsymbol{R}_{\boldsymbol{\omega}} \cdot \frac{1}{M^{i+1}} \cdot \boldsymbol{I}_{\boldsymbol{i}+\mathbf{1}}-\boldsymbol{R}_{\boldsymbol{\omega}} \cdot \frac{1}{\boldsymbol{M}^{i}} \cdot \boldsymbol{I}_{\boldsymbol{i}}
$$

thus we have the correponding design matrix

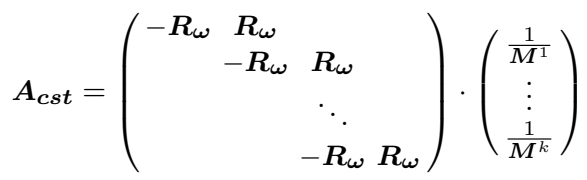

Attribute to the different mapping functions $\boldsymbol{M}$, when $\boldsymbol{A}_{\boldsymbol{c s t}}$ is defined as Eq. (27), Eq. (25) does not longer hold and the zenith ionosphere delay $\boldsymbol{I}_{\boldsymbol{z}}$ is estimable. However, it should be emphasized that the zenith ionospheric delays over a single station can be hardly considered as belonging to a second-order stationary process 
because it is subject to diurnal variation. More importantly, it usually takes a long time for the mapping function to be apparently different, in which case, the zenith ionosphere delay between the adjacent epochs is less correlated. Thus, only very relax constrains can be applied, which implies that the zenith ionosphere delay estimate may still not be sensitive to its absolute value.

\subsection{DESIGN}

As the GTSF approach represents the ionosphere delay with only deterministic characteristic, the problem is, that those function constrains may not follow the irregular spatial and temporal variations. On the contrary, the SIP approach represents the method with only stochastic characteristic and the ionosphere terms turn out to be non-estimable or not sensitive to its absolute value. Therefore, [Shi et al. (2012)] proposed the DESIGN (Deterministic plus Stochastic Ionosphere model for GNSS) approach

$$
I_{z}=a_{0}+a_{1} d L+a_{2} d L^{2}+a_{3} d B+a_{4} d B^{2}+r_{r}^{s}
$$

while $a_{0}$ is the average value of ionosphere delay over the station; $a_{1}, a_{2}$ and $a_{3}$, $a_{4}$ are the coefficients of the two second-order polynomials along east-west and south-north direction, respectively; $r_{r}^{s}$ is the SIP for each satellite; $d L\left(\begin{array}{l}s \\ r\end{array}\right), d B\left(\begin{array}{l}s \\ r\end{array}\right)$ are the longitude and latitude differences between the IPP and the approximate location of station, respectively. Obviously, the mismodeling effect of the twoorder polynomial function would be removed by the SIP, while, by detrending the ionospheric delay with the polynomial function, the SIP can be safely regarded as belonging to a second-order stationary process with more tight constraint applied.

Though the temporal constraint of the SIP $r_{r}^{s}$ has already been elaborately established by [Shi et al. (2012)], the deterministic part expressed by a secondorder polynomial is casually treated as piece-wise constant over 5 minutes. And recalling the discussion in section 3.2 , it is argued that since $a_{i}$ is only correlated within 5 minutes, the absolute ionospheric delay can hardly be estimated stably as only slow change is experienced for these coefficients. Thus in this section, the DESIGN model is further improved by a systematical study on the temporal behavior of $a_{i}$ based on the methodology of Fourier transform, and update the stochastic model of $r_{r}^{s}$ accordingly.

In the analysis of the following section, two years data collected at about 200 IGS stations in 2010 (low solar activity) and 2014 (high solar activity) with an interval of 30 second are utilized in the analysis of the DESIGN model. The distribution of the corresponding network is presented in Fig. 1.

\subsubsection{Deterministic part of ionosphere delay}

Theoretically, a periodic signal $f(t)$ can be expressed as the Fourier series

$$
f(t)=\alpha_{0}+\sum_{j=1}^{n}\left(\beta_{j} \cdot \sin \left(2 \pi t f_{j}\right)+\gamma_{j} \cdot \cos \left(2 \pi t f_{j}\right)\right)
$$




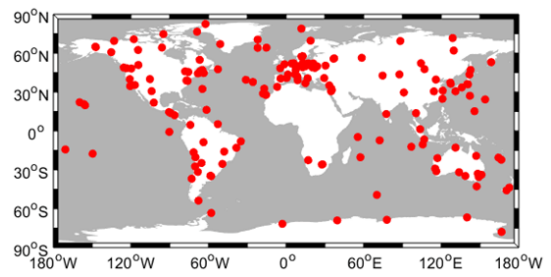

Fig. 1 Distribution of over 150 global tracking stations for the statistic study of ionosphere parameters in DESIGN

in which, $n$ is the number of the frequencies for this signal, $f_{j}$ is the $j^{\text {th }}$ frequency, $\alpha_{0}, \beta_{j}$ and $\gamma_{j}$, the amplitudes for each frequency, denoting the power of different components. Based on the Fourier analysis, the value of those terms can be determined with a set of uniform sampling.

Concerning the frequency components of $a_{i}$ in Eq. (28), samples are obtained with an interval of 5 minutes for 2010 and 2014 based on PPP [Shi et al. (2012)]. By applying Fourier analysis, the power spectrum of different frequency components for $a_{i}$ is shown in Fig. 2. As the DESIGN model is focused on the daily solution, the periodic items larger the 24 hours are removed in the plot. It is demonstrated by Fig. 2 that for both ionosphere quite and active years, though the amplitude varies, the frequency components are roughly identical for different coefficients $a_{i}, i \in\left(\begin{array}{lllll}0 & 1 & 2 & 3 & 4\end{array}\right)$ and different stations. While the main common frequency components $f_{i}$ are listed in Tab. 1 according to their amplitude. These period properties are most likely due to the joint effect of the ionosphere behavior and the satellite orbit design. Though a throughout study on the physical explanation of these frequencies is expected to further improve GNSS data processing, the subject itself is full of challenge and beyond the scope of our study.

Table 1 Main frequency components for $a_{0}, a_{1}, a_{2}, a_{3}$ and $a_{4}$ arranged according to descending amplitude

\begin{tabular}{cc}
\hline Component & Frequency $[1 /$ hour $]$ \\
\hline$f_{1}$ & $1 / 24$ \\
$f_{2}$ & $1 / 12$ \\
$f_{3}$ & $1 / 8$ \\
$f_{4}$ & $1 / 6$ \\
$f_{5}$ & $1 / 4.8$ \\
$f_{6}$ & $1 / 4$ \\
$f_{7}$ & $1 / 3.4$ \\
$f_{8}$ & $1 / 3$ \\
$f_{9}$ & $1 / 2.7$ \\
$f_{10}$ & $1 / 2.4$ \\
\hline
\end{tabular}

As suggested by Fig. 2, it is apparent that there are actually more components, especially during the ionosphere active period. It is expected that more components involved in the modeling implies an exhaustive description, while at the cost of extra computation load and the potential of numerical problems during the initialization of GNSS data processing. In our opinion, the model to be accepted 


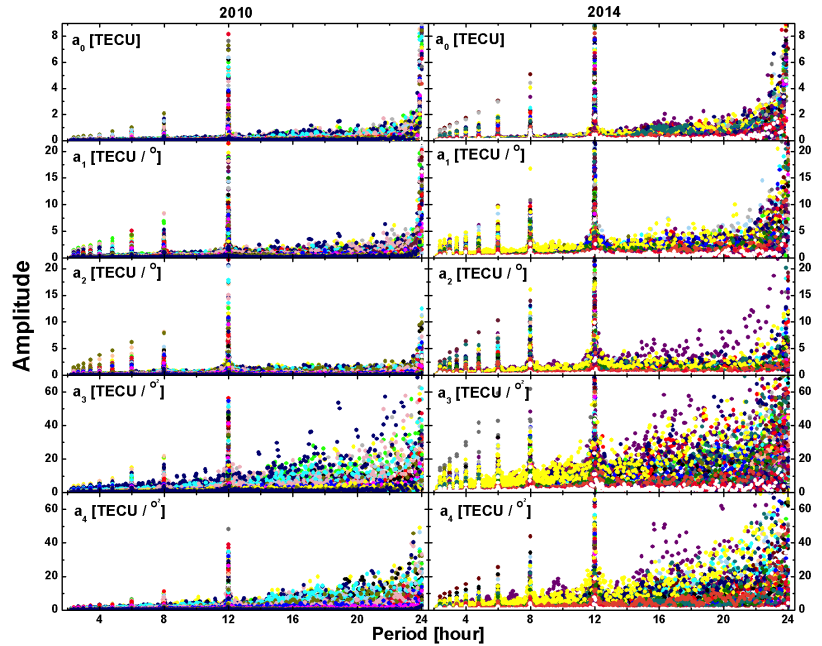

Fig. 2 Power spectrum of different frequency components for $a_{i}$ based on the Fast Fourier Transform with over 150 stations denoted in different colors for 2010 (left) and 2014 (right)

in the practical solution should not only ideally describe the ionosphere delay, but should also keep reasonably simple. Thus, in this contribution, we fix the frequency components $f_{1}=1 / 24, f 2=1 / 12$ and $f_{3}=1 / 8$, and estimate the amplitude as daily constant that updated every 24 hours. Thus, an time-related decomposition of the deterministic part of DESIGN is available as Eq. (30).

$$
a_{i}(t)=\alpha_{0}+\sum_{j=1}^{3}\left(\beta_{j} \cdot \sin \left(2 \pi t f_{j}\right)+\gamma_{j} \cdot \cos \left(2 \pi t f_{j}\right)\right)
$$

and the total number of coefficients, i.e., $\alpha_{0}, \beta_{j}$ and $\gamma_{j}$ to describe the deterministic part is $5 \cdot(1+2 n)=35$.

\subsubsection{Stochastic part of ionosphere delay}

The crucial parameter of a random process $Z(s)$ ( $s$ is the spatial and/or time variables) is its variogram $2 \gamma\left(s_{i}, s_{j}\right)$ which can be obtained based on a finite sample with a robust method as suggested by [Cressie and Hawkins (1980)]

$$
2 \hat{\gamma}(h) \equiv\left(\frac{1}{|N(h)|} \sum_{N(h)}\left|r\left(s_{i}\right)-r\left(s_{j}\right)\right|^{1 / 2}\right)^{4} /\left(0.457+\frac{0.494}{|N(h)|}\right)
$$

where $2 \hat{\gamma}(h)$ is the estimate of variogram; $r(s)$ is defined in model (28) for epoch $s$; the sum is over $N(h) \equiv\left\{(i, j): s_{i}-s_{j}=h\right\}$ and $|N(h)|$ is the number of distinct elements of $N(h)$. From definition, the variogram $2 \gamma(h)$ relates to the covariance by the following equations

$$
2 \gamma\left(s_{i}, s_{j}\right)=2\left(C(0)-C\left(s_{i}-s_{j}\right)\right)
$$




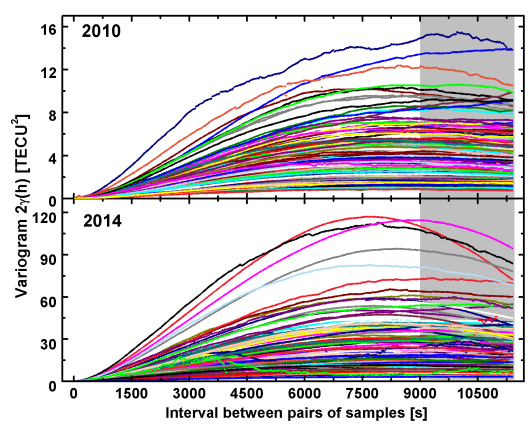

Fig. 3 Variogram of the ionospheric delay residuals $r$ for over 150 stations averaged over 2010 (upper) and 2014 (bottom). The horizontal axis represents the time interval between two samples, i.e., $h=s_{i}-s_{j}$ in Eq. (31)

with $C(\cdot)$ the covariogram which is defined by covariance directly

$$
C\left(s_{i}-s_{j}\right)=\operatorname{cov}\left(r\left(s_{i}\right), r\left(s_{j}\right)\right)
$$

Thus, the quantity variogram describes the temporal behavior of $r$ exactly. Since a rigorous mathematical background concerning variogram has been given previously by [Shi et al. (2012)], we present the statistic results directly in this section.

From the discussion in section 3.3.1, the deterministic part can be estimated and removed from Eq. (28) based on Eq. (30), then the behavior of the stochastic part, i.e., $r_{r}^{s}$ is studied based on the same sample of two years GNSS data according to Eq. (31).

Fig. 3 gives the variograms of the ionospheric delay residuals for all the stations. In general, the variogram increases with distance. With the distance increases from 0 , the variogram increases from 0 and becomes a constant as the distance is up to $9000 \mathrm{~s}$. Moreover, consistent with the previous study in [Shi et al. (2012)], the variogram increases faster against the distance for lower latitude stations, which indicates that zenith ionospheric delay residuals become less correlated for these stations.

Regarding the plot in Fig. 3, the spherical model is selected for the parametric semivariogram

$$
\gamma(h)= \begin{cases}c_{s}\left((3 / 2)\left(h / a_{s}\right)-(1 / 2)\left(h / a_{s}\right)^{3}\right) & , 0 \leq h<a_{s} \\ c_{s} & , h \geq a_{s}\end{cases}
$$

in which $a_{s}$ is the maximum correlation distance, which is about $9000 \mathrm{~s}$ as suggested by Fig. 3, while $c_{s}$ corresponds to the constant when the residuals become uncorrelated.

The experiment value of $c_{s}$ for different stations is given by Fig. 4 as gray circle. As presented, $c_{s}$ is related to geomagnetic latitude and the ionosphere activity status. To best fit these experiment values, the Gaussian function is utilized to describe the geomagnetic latitude $B$ variation, and the peak of this Gaussian function is around $15^{\circ}$ which is due to the ionosphere equator anomaly.

$$
c_{s}=c_{s, \min }+c_{s, \max } \cdot e^{-|| B|-15|^{2} / 128}
$$




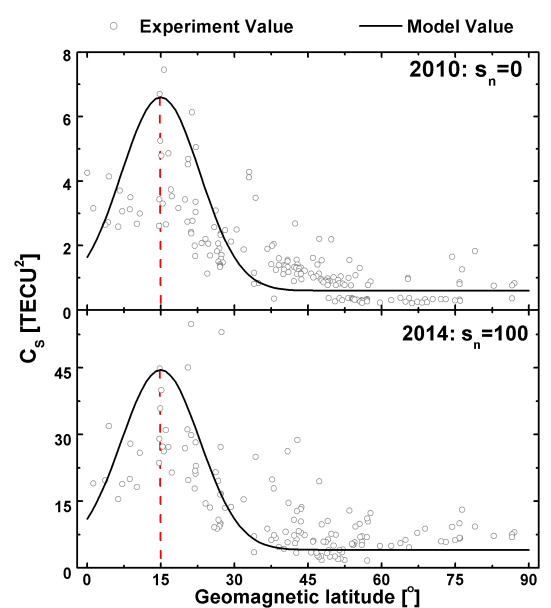

Fig. 4 Varying of $c_{S}$ against the geomagnetic latitude for 2010 (upper) and 2014 (bottom). The experiment value for different stations is denoted in gray circle, while the model value is denoted in black line, the red dashed line denotes the peak of the Gaussian function

while the variation of ionosphere activity is modeled by $c_{s, \min }$ and $c_{s, \max }$ with Epstein function as

$$
\left\{\begin{array}{l}
c_{s, \text { min }}=0.6+(7.5-0.6) \cdot\left(1 /\left(1+e^{x}\right)\right) \\
c_{s, \text { max }}=6.0+(75.0-6.0) \cdot\left(1 /\left(1+e^{x}\right)\right)
\end{array}\right.
$$

with $x$ dependent on the Sunspot number $s_{n}$

$$
x\left(s_{n}\right)=\left(s_{n}-100\right) / 20
$$

The Epstein function has also been widely used for the electron density profile modeling in International Reference Ionosphere (IRI) by [Bilitza (1992)].

Based on Eqs. (35), (36) and (37), the model value of $c_{s}$ is then presented in Fig. 4 as black line with the Sunspot number set as $s_{n}=0$ and $s_{n}=100$ for 2010 and 2014, respectively.

Furthermore, for the readers to get an intuitive graphic of the Sunspot number and $c_{s, \min }, c_{s, \max }$ based on Eqs. 36 and 37, Figs. 5 and 6 give the international Sunspot number of the last 13 years, and the serial of $c_{s, \min }$ and $c_{s, \max }$ with $s_{n}$ ranging from 0 to 200 .

\subsection{3 discussion}

Based on the observables collected by over 150 stations for two years, the temporal behavior of the deterministic part $a_{i}$ and stochastic part $r$ of DESIGN has been studied. The constrains on $a_{i}$ are expressed as Fourier series based on Eq. (30) with frequency fixed and amplitude estimated, while $r$ is estimated as the random walk with the covariance between epochs summarized by Eq. (34) to (37). Furthermore, since the prediction value of Sunspot number is available as indicated by Fig. 5, the proposed method DESIGN can be easily adapt itself to real-time applications. 


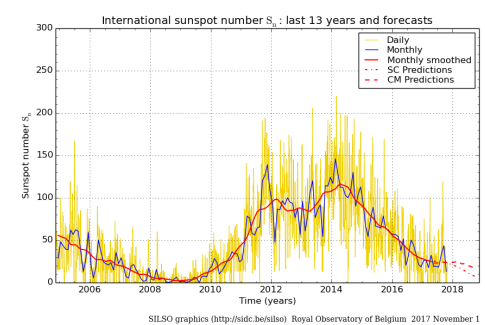

Fig. 5 International Sunspot number $s_{n}$ for the last 13 years and forecasts given by Sunspot Index and Long-term Solar Observations (SILSO, http://www.sidc.be/silso/home)

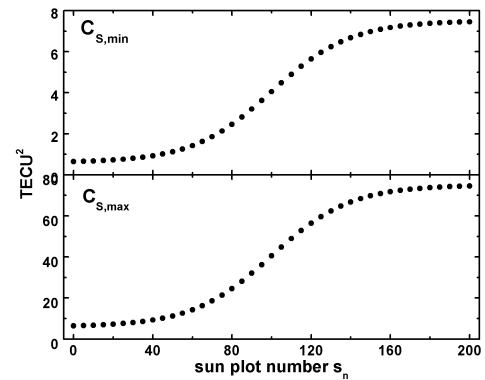

Fig. 6 Serial of $c_{s, \min }$ (upper) and $c_{s, \max }$ with respect to $s_{n}$ ranging from 0 to 200

Compared with the former work concerning DESIGN approach presented in our previous study, i.e., [Shi et al. (2012)], we go one step further by modeling the daily variation of the deterministic part with Fourier series in this contribution. Moreover, the annual variation of variogram for $r$ is derived based on the statistical analysis with both ionosphere active and quiet observable. Finally, the geomagnetic coordinate is selected in the modeling instead of the geodetic coordinate.

\section{Experimental validation}

\subsection{Data and strategy}

To cope with the upcoming or available new and modernized GNSS signals, especially, the multi-frequency data processing, real-time applications, as well as the GNSS/INS integrated navigation, we launched the FUSING (FUSing IN Gnss) project. At present, the FUSING software is capable for the precise orbit determination (POD) of GNSS, high-frequency satellite clock estimation, ionosphere and troposphere modeling as well as multi-frequency precise positioning. In order to confirm the above analysis with numerical experiment, we have adopted the novel ionosphere parameterization model, i.e., DESIGN, into the FUSING software package.

In the following demonstration, both single- and dual-frequency PPP based on the undifferenced and uncombined observable model constrained with DESIGN is 
Table 2 Details of the data

\begin{tabular}{cccc}
\hline Item & $\mathbf{2 0 1 0}$ & $\mathbf{2 0 1 4}$ & $\mathbf{2 0 1 7}$ \\
\hline DOY & $001-030$ & $335-365$ & $001-030$ \\
Station number & 85 & 53 & 72 \\
GNSS & $G$ & $G R C E$ & $G R C E$ \\
$\boldsymbol{S}_{\boldsymbol{n}}$ & 0 & 100 & 30 \\
\hline
\end{tabular}

Table 3 Details of the PPP processing strategy

\begin{tabular}{|c|c|c|}
\hline \multirow{2}{*}{ Item } & \multirow{2}{*}{$\begin{array}{l}\text { PPP-IF } \\
\text { IF }\end{array}$} & PPP-RAW \\
\hline & & DESIGN-35 \\
\hline Period & \multicolumn{2}{|c|}{ DOY 001-030, 2010; DOY 335-365, 2014; DOY 001-030, 2017} \\
\hline Observable & Ionospere free combination & Undifferenced and uncombined observable \\
\hline Frequency used & \multicolumn{2}{|c|}{ GPS: L1/L2; GLONASS: G1/G2; BDS: B1/B2; Galileo: E1/E5a } \\
\hline Solution & \multicolumn{2}{|c|}{ Daily dynamic solution in simulated real-time with square root information filter } \\
\hline Satellite orbit & \multicolumn{2}{|r|}{ Fixed with IGS final orbit products } \\
\hline Satellite clock & \multicolumn{2}{|r|}{ Fixed with IGS final clock products } \\
\hline $\mathrm{PCO} / \mathrm{PCV}$ & \multicolumn{2}{|r|}{ Corrected } \\
\hline Tides & \multicolumn{2}{|r|}{ Corrected } \\
\hline Phase windup & \multicolumn{2}{|c|}{ Corrected } \\
\hline Receiver clocks / ISBs & \\
\hline Satellite DCB & \multicolumn{2}{|c|}{$\begin{array}{l}\text { One clock for each system as white noise parameter, and no ISBs are involved } \\
\text { Absorbed into satellite clock } \\
\text { Fixed with IGS DCB products }\end{array}$} \\
\hline Receiver DCB & \multicolumn{2}{|c|}{ Absorbed into receiver clock $\quad$ Estimated as constant } \\
\hline Troposphere & \multicolumn{2}{|c|}{ Prior model with remaining estimated as a random walk process } \\
\hline Ionosphere & \multirow[t]{2}{*}{ Eliminate the first order effect } & Constrained as [Shi et al. (2012)] Constrained as section 3.3 \\
\hline Ambiguity & \\
\hline Elevation angle cutoff & & \\
\hline Weighting & & \\
\hline Reference & \multicolumn{2}{|c|}{$\frac{3 \mathrm{dm} \text { for pseudo-range and } 3 \mathrm{~mm} \text { for phase, low elevation observables and outliers are down-weighted }}{\text { Daily static solution with PANDA }}$} \\
\hline Statistic & \multicolumn{2}{|c|}{$\begin{array}{l}\text { Daily static solution with PANDA } \\
\text { Based on the whole daily session, i.e., positioning of both before and after convergence are included }\end{array}$} \\
\hline
\end{tabular}

Table 4 Total ionosphere parameter number of 24 hours with an interval of $30 \mathrm{sec}$

\begin{tabular}{lccc}
\hline Parameter & IF & DESIGN-5 & DESIGN-35 \\
\hline Deterministric & 0 & $5 \cdot 288^{a}$ & 35 \\
Stochastic & 0 & $n^{b} \cdot 2880$ & $n \cdot 2880$ \\
Total Number & 0 & $1440+n \cdot 2880$ & $35+n \cdot 2880$ \\
\hline$a$ The ionosphere deterministric parameters are updated \\
every 5 minutes in DESIGN-5 \\
$b$ The average number of available satellites for each epoch
\end{tabular}

carried out and assessed in terms of accuracy and convergence. Taking the dependence of DESIGN on the ionosphere activity status into consideration, observable of 2010, 2014 and 2017 are collected for the experiment with one month data for each year as presented in Tab. 2. It is noted that the Sunspot number for these years are 0,100 and 30 , respectively. The summary of strategy is stated in Tab. 3 . As we can see, the traditional method with IF combination as well as the undifferenced and uncombined observable model constrained with the former DESIGN as introduced in our previous study [Shi et al. (2012)] are also performed for comparison purpose. For the convenience of description, these approaches are denoted as IF, DESIGN-5 and DESIGN-35, respectively in the following discussion, and 
the main difference of these approaches are the processing strategy of DCB and ionosphere parameters. Meanwhile, the total ionosphere parameter number of 24 hours for different methods is presented in Tab. 4.

\subsection{PPP}

The position accuracy is measured by the differences between the estimated coordinates and the reference coordinates from the daily static solution with PANDA. Daily positioning RMS , together with the mean bias between the estimated coordinates and the reference coordinates in horizontal and vertical are derived for all the stations. Then the daily RMS and the absolute bias with respected to the reference coordinate for each epoch are obtained. Presented in Tab. 5 is the mean RMS and bias that averaged over all the samples for each year. Overall, the performance with undifferenced and uncombined PPP based on DESIGN performs better than that of traditional IF PPP. And the result of the comparison between IF and DESIGN-5 is consistent with that of [Shi et al. (2012)] and [Lou et al. (2015)], in which, only slight improvement is achieved for dual frequency PPP, while the improvement for single frequency PPP is rather significant. Moreover, as evidenced by the result of DESIGN-5 and DESIGN-35, the precision can be further improved by refining the parameterization strategy of DESIGN as introduced in section 3.3. Concerning the RMS results in dual frequency PPP, the averaged 3D improvement of DESIGN-35 is $17.8 \%$ and $7.6 \%$ compared with IF and DESIGN-5, respectively. While, for single frequency PPP, the averaged 3D improvement of RMS is $37.0 \%$ and $14.5 \%$ compared with IF and DESIGN-5, respectively.

Table 5 Experimental statistics for single- and dual-frequency PPP

\begin{tabular}{cccccccccc}
\hline & & \multicolumn{3}{c}{ Dual Frequency [cm] } & \multicolumn{3}{c}{ Single Frequency [cm] } \\
& & Vertical & \multicolumn{2}{c}{ Horizontal } & \multicolumn{2}{c}{ Vertical } & \multicolumn{2}{c}{ Horizontal } \\
& & RMS & Bias & RMS & Bias & RMS & Bias & RMS & Bias \\
\hline \multirow{2}{*}{$\mathbf{2 0 1 0}$} & IF & 15.5 & 1.4 & 10.2 & 6.7 & 48.7 & 17.5 & 33.3 & 10.5 \\
& DESIGN-5 & 9.3 & 0.7 & 7.3 & 2.7 & 28.7 & 5.4 & 20.1 & 8.1 \\
& DESIGN-35 & 9.5 & 0.9 & 7.3 & 2.7 & 30.2 & 5.3 & 19.9 & 6.9 \\
\hline \multirow{2}{*}{$\mathbf{2 0 1 4}$} & IF & 15.6 & 2.0 & 10.9 & 3.7 & 53.9 & 11.3 & 41.4 & 9.2 \\
& DESIGN-5 & 15.0 & 2.3 & 11.9 & 3.1 & 38.8 & 11.3 & 29.7 & 9.0 \\
& DESIGN-35 & 14.2 & 2.0 & 10.3 & 3.3 & 34.2 & 10.0 & 23.8 & 8.3 \\
\hline \multirow{2}{*}{$\mathbf{2 0 1 7}$} & IF & 8.7 & 1.2 & 5.1 & 1.4 & 35.9 & 7.9 & 23.8 & 8.6 \\
& DESIGN-5 & 9.5 & 1.3 & 6.4 & 1.5 & 34.9 & 7.0 & 22.1 & 7.7 \\
& DESIGN-35 & 8.4 & 1.2 & 5.0 & 1.2 & 24.3 & 7.2 & 16.2 & 4.1 \\
\hline
\end{tabular}

From the comparison among different years, it is noted that PPP with DESIGN35 gives the best performance in all the years, except that DESIGN-5 has a slightly better performance in 2010. This is reasonable since the ionosphere parameter constrains in DESIGN-5 are derived based on the samples exactly in 2010, while for DESIGN-35 presented in the contribution, we try to best fit the statistic result for different years under different ionosphere activity status. For the same reason, the horizontal accuracy of PPP based on DESIGN-5 in 2014 and 2017 is almost poorer than that of IF PPP, which implies the ionosphere in 


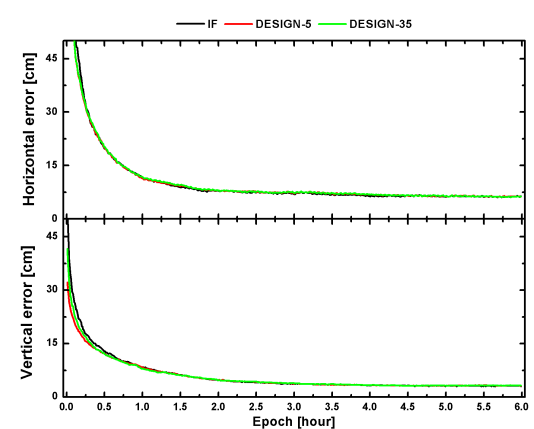

Fig. 7 Dual frequency PPP convergence serial in $68 \%$ confidence level for DOY 001-030, 2010 over the first 6 hours of horizontal (upper panel) and vertical (bottom panel), respectively

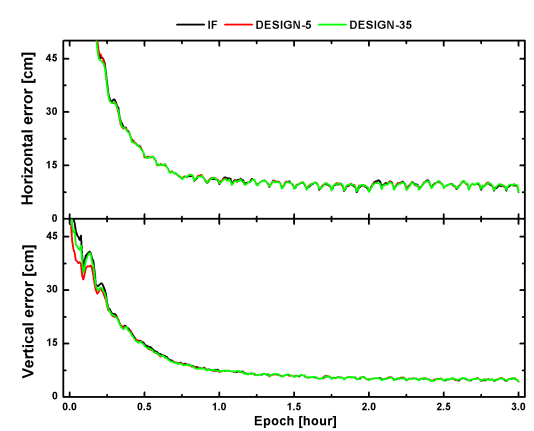

Fig. 8 Dual frequency PPP convergence serial in 68\% confidence level for DOY 335-365, 2014 over the first 6 hours of horizontal (upper panel) and vertical (bottom panel), respectively

2014 and 2017 may over constrained by DESIGN-5. In addition, regardless the PPP approach involved, benefitting from the increasing number of multi-GNSS satellites as well as the improved precise ephemeris, the PPP result in 2017 is apparently better than that of 2010 and 2014 .

Besides the RMS presented in Tab. 5, Fig. 7 to Fig. 9 further give the averaged dual frequency PPP convergence serial during the initialization for different years. As we can see, the performances are roughly identical with different approaches, except during the first hour of 2017, in which it takes a longer initialization time for DESIGN-5. This is because that the ionosphere constrain of DESIGN-5 is based on GPS observable for 2010 only, which is not suitable for multi-GNSS data processing under different ionosphere activity status. In addition, it is noted that there is a 5 minutes periodic term in 2014 as illustrated in Fig. 8, especially for the horizontal component. It is most likely attributed to the imperfect multi-GNSS satellite clock products with a resolution of 5 minutes.

Similarly, shown in Fig. 10 to Fig. 12 is the convergence serial corresponding to single frequency PPP. From which it is concluded, that in all the cases the green line, i.e., result based on DESIGN-35 performs obviously better than the traditional IF and previous approaches both, before and after convergence. Fur- 


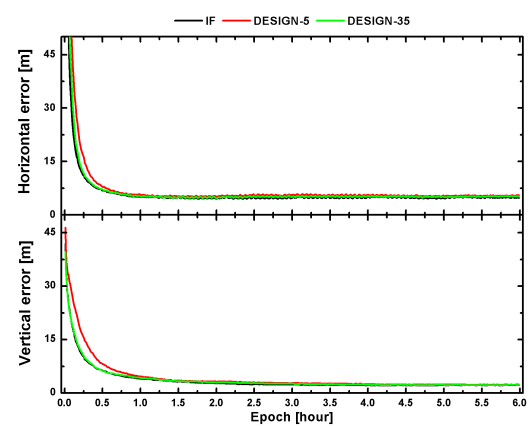

Fig. 9 Dual frequency PPP convergence serial in 68\% confidence level for DOY 001-030, 2017 over the first 6 hours of horizontal (upper panel) and vertical (bottom panel), respectively

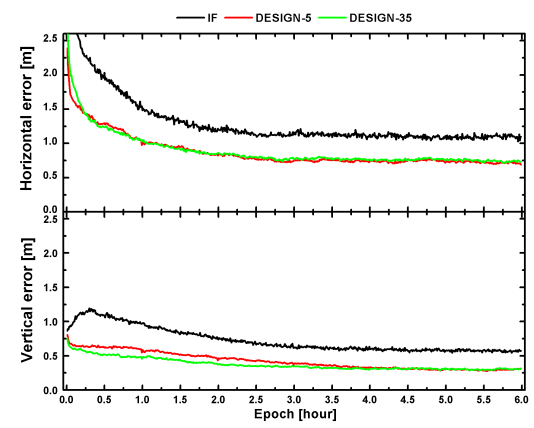

Fig. 10 Single frequency PPP convergence serial in 68\% confidence level for DOY 001-030, 2010 over the first 6 hours of horizontal (upper panel) and vertical (bottom panel), respectively

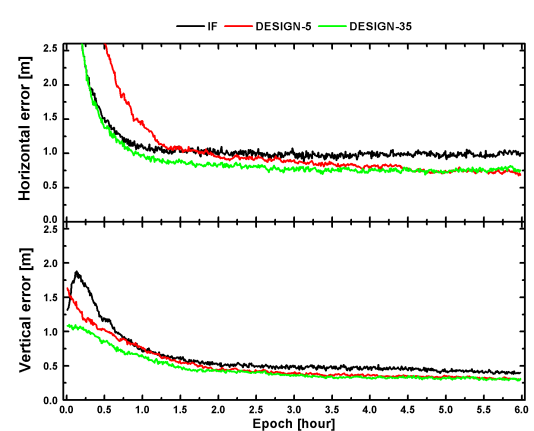

Fig. 11 Single frequency PPP convergence serial in $68 \%$ confidence level for DOY 335-365, 2014 over the first 6 hours of horizontal (upper panel) and vertical (bottom panel), respectively 


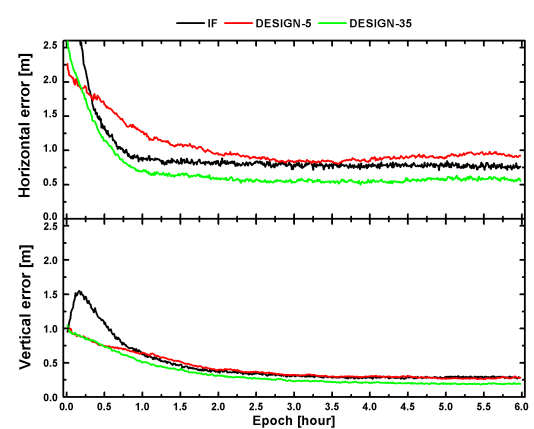

Fig. 12 Single frequency PPP convergence serial in $68 \%$ confidence level convergence serial for DOY 001-030, 2017 over the first 6 hours of horizontal (upper panel) and vertical (bottom panel), respectively

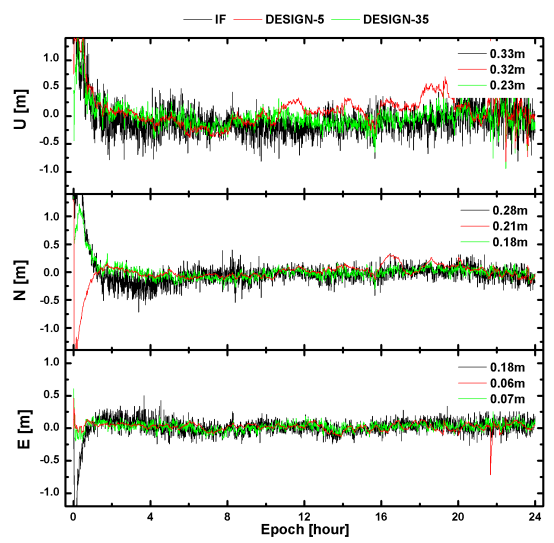

Fig. 13 Single frequency PPP serial in the Up (U), North (N) and East (E) directions for station NKLG on DOY 001, 2017.

thermore, Fig. 13 presents the single frequency kinematic PPP time serial in DOY 001, 2017 for station NKLG. As shown in Fig. 13, the noisiest series is derived with IF PPP, since the single-frequency IF combination is highly dependent on pseudorange observable. In addition, though DESIGN-5 PPP gives the smoothest result, the best PPP precision is obtained by DESIGN-35, which again confirms that the ionosphere delay may over constrained by DESIGN-5, i.e., the model derived under the quiet ionosphere activity status.

\section{Conclusions}

The ionosphere delay is one of the critical issues of the undifferenced and uncombined observable GNSS data processing model. Concerning developing of the ionosphere delay parameterization method for single station GNSS data processing, the 
involving authors have promoted the embryo model of DESIGN in the previous study of [Shi et al. (2012)]. In which the ionosphere delay is decomposed into deterministic and stochastic component, while the deterministic part expressed with second-order polynomial is estimated as piece-wise constant over 5 minutes and the stochastic part is estimated as random walk with constrains derived based on statistic of 4 weeks data in 2010 .

In this contribution, we further model the daily variation of deterministic part with Fourier series and update the variogram of the stochastic part accordingly based on two year data collected by about 150 stations. From the statistic studies, it is concluded that the main frequency components are identical for different coefficients $a_{i}, i \in\left(\begin{array}{llll}0 & 1 & 2 & 3\end{array} 4\right)$, different stations, as well as different ionosphere activity status, but with varying amplitude. Thus, in the Fourier series expression of deterministic part, we fixed the frequency and estimated the amplitude as daily constant unknowns. Concerning the stochastic component, the variation of the variogram is both, geomagnetic latitude and ionosphere activity status dependent. Thus, we use the Gaussian function and Epstein function to model the variation of geomagnetic latitude and ionosphere activity status, respectively.

The updated DESIGN, i.e., DESIGN-35 is then adopted in undifferenced and uncombined observable PPP for both dual frequency and single frequency, and compared with the traditional IF PPP, i.e., IF and undifferenced and uncombined PPP with formal DESIGN, i.e., DESIGN-5. Three months data collected in 2010, 2014 and 2017 are utilized in the experiment demonstration. The results suggest that for dual frequency PPP, the averaged 3D improvement of DESIGN-35 is $17.8 \%$ and $7.6 \%$ compared with IF and DESIGN-5, respectively. While, for single frequency PPP, the averaged 3D improvement is $37.0 \%$ and $14.5 \%$ compared with IF and DESIGN-5, respectively.

To access the full capabilities of the modernized and newly deployed multiGNSS signals, the undifferenced and uncombined observable processing model constrained with DESIGN provides a possible solution as demonstrated in the PPP applications for different ionosphere activity status. Actually, this algorithm is served as the most basic model in our GNSS data processing, e.g., PPP, ionosphere / troposphere delay estimation. It is also expected to be applied in precise orbit determination (POD) as well as satellite clock estimation (SCE), and other cases.

\section{Acknowledgements}

This study is partially sponsored by the National Key Research and Development Plan (No. 2016YFB0501802), and partially sponsored by National Natural Science Foundation of China (41504028, 41774035, 41574030, 41231174). The authors thank the anonymous reviewers for their valuable comments. Thanks also go to IGS for data provision.

\section{References}

[Baarda (1981)] Baarda W (1981) S-transformations and criterion matrices. Netherlands Geodetic Commission, Delft

[Blaha (1982)] Blaha G (1982) Free networks: minimum norm solution as obtained by the inner adjustment constraint method. Journal of Geodesy. 56(3):209-219 
[Bilitza (1992)] Bilitza D (1992) International reference ionosphere (1990). Planetary and Space Science, 40(4), 544. http://doi.org/10.1016/0032-0633(92)90174-M

[Blanch J (2003)] Blanch J (2003) Using Kriging to bound satellite ranging errors due to the ionosphere. Ph.D. Thesis, Stanford University, California

[Bock et al. (2009)] Bock H, Jäggi A, Dach R, Schaer S, Beutler G (2009) GPS singlefrequency orbit determination for low Earth orbiting satellites. Adv Space Res. 43(5):783-791, doi:10.1016/j.asr.2008.12.003

[Chen and Gao (2005)] Chen K, Gao Y (2005) Real-Time Precise Point Positioning Using Single Frequency Data, in: Proceedings of the 18th International Technical Meeting of the Satellite Division of The Institute of Navigation. The Institute of Navigation, Inc., Fairfax, USA, pp. 1514-1523

[Cressie and Hawkins (1980)] Cressie N, Hawkins DM (1980) Robust estimation of the variogram: I. Mathematical Geology. 12(2), 115-125, doi:10.1007/BF01035243, 1980

[Feltens and Schaer(1998)] Feltens J, Schaer S (1998) IGS products for the ionosphere. In: Proceedings of the 1998 IGS analysis centers workshop, ESOC,Darmstadt, Germany

[Gu (2013)] Gu S (2013) Research on the Zero-difference Un-combined Data Processing Model for Multi-frequency GNSS and Its Applications. PhD, Wuhan University (In Chinese).

[Gu et al. (2013)] Gu S, Shi C, Lou Y, Feng Y, Ge M (2013) Generalized-Positioning for Mixed-Frequency of Mixed-GNSS and Its Preliminary Applications. China Satellite Navigation Conference (CSNC) 2013 Proceedings: 399-428

[Hernández-Pajares et al. (2009)] Hernández-Pajares M, Juan JM, Sanz J, Orus R, GarciaRigo A, Feltens J, Komjathy A, Schaer S, Krankowski A (2009) The IGS VTEC maps: a reliable source of ionospheric information since 1998. Journal of Geodesy. 83(3):263-275. doi:10.1007/s00190-008-0266-1

[Kouba and Héroux (2001)] Kouba J, Héroux P (2001) Precise point positioning using IGS orbit and clock products. GPS Solut, 5(2):12-28

[Li et al. (2012)] Li Z, Yuan Y, Li H, Ou J, Huo X (2012) Two-step method for the determination of the differential code biases of COMPASS satellites. J Geodesy, 86(11):1059-1076. doi: $10.1007 / \mathrm{s} 00190-012-0565-4$

[Lou et al. (2015)] Lou Y, Zheng F, Gu S, Wang C, Guo H, Feng Y (2015) Multi-GNSS precise point positioning with raw single-frequency and dual-frequency measurement models. GPS Solutions, 20(4), 849-862. http://doi.org/10.1007/s10291-015-0495-8

[Rao and Mitra (1971)] Rao CR, Mitra SK (1971) Generalized inverse of a matrix and its applications. New York.

[Schaer (1999)] Schaer S (1999) Mapping and predicting the Earth's ionosphere using the Global Positioning System. Ph.D. Thesis, University of Bern, Switzerland

[Schönemann et al. (2011)] Schönemann E, Becker M, Springer T (2011) A new approach for GNSS analysis in a multi-GNSS and multi-signal environment. Journal of Geodetic Science, 1(3):204-214. doi:10.2478/v10156-010-0023-2

[Shi et al. (2016)] Shi C, Fan L, Li M, Liu Z, Gu S, Zhong S, Song W (2016) An enhanced algorithm to estimate BDS satellite's differential code biases. Journal of Geodesy, 90(2), 161?177. http://doi.org/10.1007/s00190-015-0863-8

[Shi et al. (2012)] Shi C, Gu S, Lou Y, Ge M (2012A) An improved approach to model ionospheric delays for single-frequency Precise Point Positioning. Adv Space Res, 49(12):16981708. doi:10.1016/j.asr.2012.03.016

[Shi et al. (2012B)] Shi C, Zhao Q, Li M, Tang W, Hu Z, Lou Y, Zhang H, Niu X, Liu J (2012B) Precise orbit determination of Beidou Satellites with precise positioning. SCIENCE CHINA Earth Sciences. 55(7):1079-1086. doi:10.1007/s11430-012-4446-8

[Welsch (1979)] Welsch W (1979) A review of the adjustment of free networks. Survey Review. 25(194), 167-180

[YiChung (1997)] YiChung C (1997) Real Time Implementation of the Wide Area Augmentation System for the Global Positioning System with an Emphasis on Ionospheric Modeling. PhD., Stanford University

[Yuan and $\mathrm{Ou}(2004)$ ] Yuan Y and $\mathrm{Ou} \mathrm{J} \mathrm{(2004)} \mathrm{A} \mathrm{generalized} \mathrm{trigonometric} \mathrm{series} \mathrm{function}$ model for determining ionospheric delay. Progress in Natural Science, 14(11):1010-1014

[Zumberge et al. (1997)] Zumberge JF, Heflin MB, Jefferso DC, Watkins MM, Webb FH (1997) Precise point positioning for the efficient and robust analysis of GPS data from large networks. J Geophys Res. 102(B3):5005-5017 